\title{
Disentangling the Role of Shape, Ligands, and Dielectric Constants in the Absorption Properties of Colloidal CdSe/CdS Nanocrystals
}

\author{
Ilaria Angeloni, ${ }^{\dagger \dagger}$ Waseem Raja, ${ }^{\dagger, \S}$ Rosaria Brescia, ${ }^{\dagger}$ Anatolii Polovitsyn, ${ }^{\dagger, \S}$ Francesco De Donato, ${ }^{\dagger}$ \\ Maurizio Canepa, ${ }^{\S}$ Giovanni Bertoni, ${ }^{\dagger, \|}$ Remo Proietti Zaccaria, ${ }^{\dagger}$ and Iwan Moreels ${ }^{*},{ }^{\dagger}$ \\ ${ }^{\dagger}$ Istituto Italiano di Tecnologia, via Morego 30, IT-16163 Genova, Italy \\ ${ }^{\ddagger}$ Dipartimento di Chimica and ${ }^{\S}$ Dipartimento di Fisica, Università di Genova, Via Dodecaneso 33, IT-16146 Genova, Italy \\ "IMEM-CNR, Parco Area delle Scienze, 37/A, IT-43124 Parma, Italy
}

\section{Supporting Information}

\begin{abstract}
We calculated the absorption coefficient of colloidal wurtzite $\mathrm{CdSe} / \mathrm{CdS}$ nanocrystals (NCs), ranging from spherical to strongly elongated shapes (aspect ratio up to 21) by correlating the NC absorbance spectrum to the NC elemental composition and overall dimensions. We compared experimental data with numerical finite-element calculations of the NC absorption coefficient, which can account for the nonspheroidal NC shape and the influence of the organic ligand shell. The results unveiled that quantum confinement

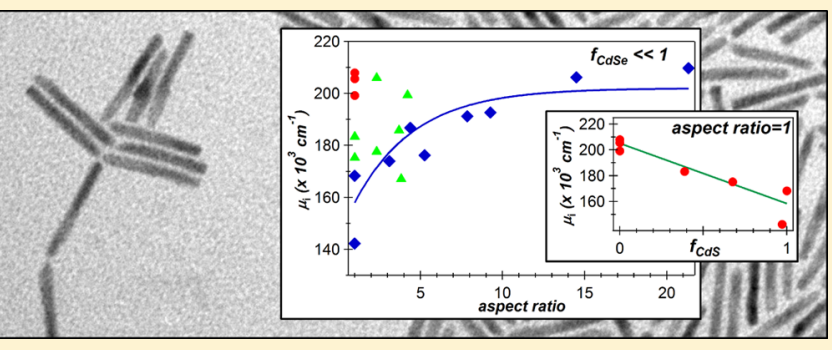
effects influence the NC absorption properties, even at relatively high photon energies $(\sim 4.2 \mathrm{eV})$. From a practical point of view, we provide a general expression for the absorption coefficient that only requires the knowledge of the $\mathrm{NC}$ aspect ratio and $\mathrm{CdSe} / \mathrm{CdS}$ volume ratio, giving access to a fast and nondestructive estimate of NC concentrations in solution and single-particle absorption cross sections, relevant for a wide range of photonic applications. More fundamentally, the unique aspect ratio-dependence of the absorption coefficient allowed us to derive the real $\left(\varepsilon_{\mathrm{r}}\right)$ and imaginary $\left(\varepsilon_{\mathrm{i}}\right)$ parts of the dielectric constant, demonstrating that even at energies far above the band gap the NC absorption coefficient differs from bulk due to a reduction of both $\varepsilon_{\mathrm{r}}$ and $\varepsilon_{\mathrm{i}}$. As the methods presented are general, our analysis can be applied to a wide range of materials of varying composition and yields comprehensive insight into the optical constants of colloidal nanocrystals.
\end{abstract}

KEYWORDS: absorption coefficient, molar extinction coefficient, absorption cross section, nanorods, quantum dots,

Maxwell-Garnett effective medium approximation

$\mathrm{V}$ ersatile and low-cost synthesis routes make colloidal semiconductor nanocrystals (NCs) frontier materials for optoelectronic and photonic applications. They produce NCs with bright fluorescence and narrow emission line widths that cover a broad spectral range, and with decay times tunable from nanoseconds to microseconds. ${ }^{1-5}$ Heterostructured NCs (hNCs) consisting of a core encapsulated by a second semiconductor create the possibility to further engineer the electron and hole wave function overlap by tuning the conduction band (CB) and valence band (VB) alignment from type I to type II. ${ }^{1,2}$ Among the various systems, $\mathrm{CdSe}$ (core)/CdS(shell) hNCs are arguably the most prominent. Spherical CdSe/CdS hNCs (dot-in-dots, DIDs) started drawing interest almost two decades ago, with substantial synthesis optimizations reported toward improved size control, ${ }^{6}$ near-unity photoluminescence quantum efficiency (PL QE), ${ }^{7}$ and strongly suppressed PL intermittency (blinking). ${ }^{8,9}$ The shallow conduction band offset leads to an electron that, being only bound to the hole via Coulomb interactions, delocalizes into the CdS shell. This identifies these structures as quasi-type II hNCs, with "giant-shell" $\mathrm{CdSe} / \mathrm{CdS}$
hNCs characterized by strongly reduced Auger recombination rates $^{10,11}$ and highly efficient biexciton emission ${ }^{12,13}$ as the major achievements associated with this behavior. The CdSe core can also be embedded in an elongated CdS shell, ${ }^{14}$ which enables to obtain hNCs with a mixed OD-1D dimensionality (dot-in-rods, DIRs). hNCs can be synthesized with PL QE up to $80 \%{ }^{15,16}$ and a wide range of aspect ratios. In addition to the advantages linked to the electron delocalization already observed in spherical hNCs, this system yields linearly polarized fluorescence and further control over the linear and nonlinear absorption properties, ${ }^{17}$ excited state carrier dynamics, ${ }^{18}$ and stimulated emission ${ }^{19}$ that, as a consequence, can be triggered from either core or shell states.

Because of these characteristics, spherical and anisotropic $\mathrm{CdSe} / \mathrm{CdS}$ hNCs are currently the object of an intense research activity aimed at applying them in photonic devices such as LEDs, $^{20}$ lasers, ${ }^{19}$ nonlinear materials, ${ }^{17,21}$ and quantum emitters. ${ }^{22,23}$ For many applications, a detailed knowledge of

Received: August 12, 2015

Published: December 1, 2015 
the materials linear optical properties is required. For instance, single-exciton gain critically relies on the accurate knowledge of the single-particle absorption cross-section, ${ }^{24}$ while nonlinear optical properties and excited-state dynamics such as the shellto-core carrier relaxation are often calibrated by the linear absorption coefficient. ${ }^{25}$ However, while the absorption coefficients of core-only spherical and rod-like CdSe and CdS NCs have been reported, ${ }^{21,26-28}$ a comprehensive study incorporating the different effects from core/shell geometry, the anisotropic shape and, in the case of wurtzite (WZ) NCs, using anisotropic optical constants is presently still lacking.

The first comparisons of the NC absorption, both in suspension and glass matrices, with bulk were reported nearly 30 years ago, ${ }^{29,38}$ showing that the shape of the NC absorption spectra becomes similar to the smooth, featureless bulk absorption when the NC diameter increases. Through a local field correction of the bulk absorption, ${ }^{31}$ NC spectra were shown to converge at high energies to the spectrum of bulk semiconductors. ${ }^{32}$ More recently, quantitative studies were conducted through an independent measurement of the NC concentration in solution, with the highest accuracy obtained when the NC nonstoichiometry was taken into account. ${ }^{33,27}$ Surprisingly, this finally unveiled residual quantum confinement in Cd chalcogenide NCs, ${ }^{34,35}$ even at energies well above the band gap.

In this work, we examined the absorption coefficient of spherical and elongated $\mathrm{CdSe} / \mathrm{CdS} \mathrm{hNCs}$, with varying core-toshell volume ratios and aspect ratios from 1 to 21 , in the Maxwell Garnett effective medium approximation (MG EMA). We give a general expression for the absorption of core/shell $\mathrm{CdSe} / \mathrm{CdS} \mathrm{hNCs}$, including the influence of $\mathrm{CdS}$ volume fraction and overall aspect ratio. This yields a straightforward calculation of the NC concentration via the Beer-Lambert law, as well as the fundamental optical properties of $\mathrm{CdSe} / \mathrm{CdS}$ hNCs with different size, composition, and shape. Furthermore, through the available hNCs aspect ratios, we could make a detailed comparison with finite-element calculations of the electric field inside and near the hNCs and associated absorption coefficient. We found that the effect of nonspheroidal shape is minimal, yet the additional dielectric mismatch introduced by the organic ligand shell plays a shapedependent role. However, small differences between experiment and theory remained when using bulk optical constants of $\mathrm{CdSe}^{36}$ and $\mathrm{CdS},{ }^{37}$ which we attribute to residual confinement effects near the $E_{1}$ transition in the band structure. As the unique aspect ratio-dependence of the absorption coefficient yields independent values in both spherical (aspect ratio $=1$ ) and cylindrical (aspect ratio $=\infty$ ) limits, we could calculate the real $\left(\varepsilon_{\mathrm{r}}\right)$ and imaginary $\left(\varepsilon_{\mathrm{i}}\right)$ parts of the dielectric constant of the NCs and confirm a reduction of both $\varepsilon_{\mathrm{r}}$ and $\varepsilon_{\mathrm{i}}$ with respect to bulk optical constants.

\section{MATERIALS AND METHODS}

Materials. Trioctylphosphine oxide (TOPO, 99\%), trioctylphosphine (TOP, 97\%), tri- $n$-butylphopshine (TBP, 99\%, 10 wt \% in hexane), oleylamine (70\%), sulfur (99\%), selenium (Se, 99.99\%), and cadmium oxide (CdO, 99.999\%) were purchased from Strem Chemicals. Octadecylphosphonic acid (ODPA, 99\%) and hexylphosphonic acid (HPA, 99\%) were purchased from Polycarbon Industries. Toluene and methanol (anhydrous, for analysis) were purchased from Carlo Erba. Ethanol $(\geq 99.8 \%)$ and hexamethyldisilathiane $\left((\mathrm{TMS})_{2} \mathrm{~S}\right.$, synthesis grade) were purchased from Sigma-Aldrich.
Synthesis. To synthesize CdSe NCs, TOPO (3.0 g), ODPA $(0.28 \mathrm{~g})$, and $\mathrm{CdO}(0.06 \mathrm{~g})$ were mixed in a $50 \mathrm{~mL}$ flask and heated to about $150{ }^{\circ} \mathrm{C}$ under vacuum for $1 \mathrm{~h}$. Then the system was purged with nitrogen and heated to above $300{ }^{\circ} \mathrm{C}$ to dissolve the $\mathrm{CdO}$ until a clear and colorless mixture was obtained. At this point, $1.5 \mathrm{~g}$ of TOP and $0.5 \mathrm{~g}$ of oleylamine were inserted into the flask, the temperature was adjusted to the required injection temperature, and a Se:TOP solution $(0.058 \mathrm{~g}$ of Se with $0.360 \mathrm{~g}$ of TOP) was injected. The injection temperature and the reaction time were modified in order to synthesize CdSe NCs of different sizes. As an example, for the synthesis of green fluorescent CdSe NCs $\left(\lambda_{\mathrm{abs}}=527 \mathrm{~nm}\right)$ the Se:TOP solution was injected at $380{ }^{\circ} \mathrm{C}$ and the reaction mixture was cooled immediately after injection. Red fluorescent CdSe NCs $\left(\lambda_{\mathrm{abs}}=613 \mathrm{~nm}\right)$ were synthesized by injecting Se:TOP at $370{ }^{\circ} \mathrm{C}$ and growing the NCs for $3 \mathrm{~min}$. After synthesis, the NCs were precipitated with methanol, purified by repeated redispersion in toluene and precipitation with methanol, and finally dispersed in toluene.

A second set of CdSe NCs was synthesized according to the procedure of Carbone et al., ${ }^{15}$ with different sizes obtained by varying the growth time at $380{ }^{\circ} \mathrm{C}$. For example, the reaction time for green CdSe NCs $\left(\lambda_{\mathrm{abs}}=551 \mathrm{~nm}\right)$ was around $10 \mathrm{~s}$, while red CdSe NCs $\left(\lambda_{\text {abs }}=622 \mathrm{~nm}\right)$ were obtained in $1.5 \mathrm{~min}$ at $380{ }^{\circ} \mathrm{C}$. After growth, the flask was allowed to cool, and when the reaction mixture reached $100{ }^{\circ} \mathrm{C}, 3 \mathrm{~mL}$ of toluene was added to avoid solidification of TOPO. Finally, the NCs were precipitated by addition of methanol, followed by centrifugation and redispersion in toluene. This was repeated three times to purify the samples.

Both types of CdSe NCs were used to synthesize spherical core/shell $\mathrm{CdSe} / \mathrm{CdS} \mathrm{hNCs}$ according to the continuous injection procedure reported by Christodoulou et al. ${ }^{38}$ The second set of CdSe NCs also served as seeds for the core/shell growth of anisotropic rods. ${ }^{15}$ The $\mathrm{CdSe} / \mathrm{CdS}$ DIRs aspect ratio was controlled by varying the amount of CdSe cores used for the injection, or using different concentrations of the sulfur precursor.

Spherical CdS NCs were synthesized according to Carbone et al., ${ }^{15}$ heating TOPO, ODPA, and $\mathrm{CdO}$, and injecting a mixture of (TMS $)_{2} \mathrm{~S}$ and $\mathrm{TBP}$ at $320{ }^{\circ} \mathrm{C}$. The NCs were purified three times with methanol and subsequently redispersed in toluene.

Structural Characterization. The crystal structure was assessed by X-ray diffractometry (XRD) on representative samples for $\mathrm{CdSe}, \mathrm{CdS}$, and $\mathrm{CdSe} / \mathrm{CdS}$ core/shell hNCs. Samples were prepared by drop-casting a NC suspension on a miscut silicon substrate. Measurements were performed on a Rigaku SmartLab diffractometer with the X-ray source operating at $40 \mathrm{kV}$ and $150 \mathrm{~mA}$, in parallel beam geometry. With transmission electron microscopy (TEM) we evaluated size, shape, and monodispersity of the CdS NCs and CdSe/ CdS hNCs. Note that for CdSe, core diameters were determined from the spectral position of the first absorbance peak, using the calibration curve of Jasieniak et al. ${ }^{27}$ For the hNCs the average diameter and standard deviation were determined by TEM over 180-220 NCs. Samples were prepared by drop casting a dilute suspension on a carboncoated 200 mesh copper grid, and imaged with a JEOL JEM 1011 TEM equipped with a thermo-ionic source (W filament) operating at $100 \mathrm{kV}$. TEM measurements were also used to determine the hNC core $\left(f_{\text {CdSe }}\right)$ and shell $\left(f_{\mathrm{CdS}}=1-f_{\mathrm{CdSe}}\right)$ volume fractions. In one particular case of CdSe/CdS DIDs, the 
final shell was too thin for reliable determination of the total diameter; here data from the elemental analysis were used to estimate the core and shell volume ratios.

Elemental Analysis. The atomic concentration $c_{\text {at }}$ of digested NCs was determined by inductively coupled plasma optical emission spectrometry (ICP-OES). Samples were prepared in a $25 \mathrm{~mL}$ volumetric flask, drying a known amount under nitrogen flow and digesting the dry residue overnight in $2.5 \mathrm{~mL}$ of aqua regia. Prior to the measurement, the sample was diluted to a total volume of $25 \mathrm{~mL}$ with Millipore water, and vortexed for $10 \mathrm{~s}$ at $2400 \mathrm{rpm}$. An aliquot of the as-prepared sample was filtered using a PTFE filter with $0.45 \mu \mathrm{m}$ pore diameter. Measurements were carried out with a ThermoFisher ICAP 6000 Duo inductively coupled plasma optical emission spectrometer. At least two identical dilutions were prepared and measured, in order to verify the reproducibility of the data, and the average value was considered for each detected element. The total atomic concentration was then determined as the sum of these averages; for the different dilutions the measured atomic concentrations deviated by less than $3 \%$.

In the preparation and overnight storage of ICP-OES samples, volatile $\mathrm{H}_{2} \mathrm{~S}$ can form, leading to an underestimation of the sulfur concentration. Therefore, for three representative samples (i.e., high aspect ratio DIRs, low aspect ratio DIRs and CdSe NCs), we also evaluated the NC composition with energy dispersive X-ray spectra (EDS), collected from selected particles in scanning TEM (STEM) mode, by applying the Cliff-Lorimer quantification method. The samples were prepared by drop casting the (h)NC suspensions on carboncoated 200 mesh copper grids. The STEM-EDS analyses were carried out with a JEOL JEM-2200FS microscope equipped with a Schottky emitter working at $200 \mathrm{kV}$, a CEOS spherical aberration corrector of the objective lens and a Bruker Quantax 400 system with a $60 \mathrm{~mm}^{2}$ XFlash $6 \mathrm{~T}$ silicon drift detector. For the determination of the Cd:Se:S composition with EDS, the Cliff-Lorimer coefficients for the peaks used (Cd-L $\alpha, \mathrm{S}-\mathrm{K} \alpha$, and $\mathrm{Se}-\mathrm{K} \alpha$ lines) were refined based on the analysis of bulk CdSe and $\mathrm{CdS}$ powder references (purchased from Sigma-Aldrich, $>99.99 \%$ ). Following this calibration, results from ICP-OES and EDS were in good agreement, with maximal deviations within $\pm 8 \%$ (see Supporting Information). This confirms the reliability of ICP-OES for the sulfur concentration, and for further analysis we relied on ICP-OES data to obtain elemental concentrations.

Molar Extinction and Absorption Coefficient. To calculate the intrinsic (h)NC optical properties, identical amounts of NCs as used for the ICP-OES measurements were dried under nitrogen flow and dispersed in $3 \mathrm{~mL}$ of chloroform. The NC volume fractions $f$ in the suspensions used were of the order $f=10^{-5}$, ensuring that we worked in the highly diluted regime for which the MG EMA is valid. NC absorbance spectra were collected with a Varian Cary 300 UVvis spectrophotometer, using quartz cuvettes with an optical path length $L$ of $1 \mathrm{~cm}$. The absorbance $A$ was calculated as $-\log (T)$, with $T$ the sample transmittance (reflectance losses of the samples were corrected for by calibrating the data against cuvettes with pure solvent). For all analyses, we considered the average value of three dilutions. The typical error between the three measurements is about $3 \%$ at the wavelengths of interest $(310-295 \mathrm{~nm})$. From the ratio of $A$ and the atomic concentration $c_{\text {at }}$, we obtained the molar extinction coefficient $\varepsilon_{\text {at }}$ per atom (Beer-Lambert law, $\left.A=\varepsilon_{\mathrm{at}} \cdot c_{\mathrm{at}} \cdot L\right)$, which is proportional to the intrinsic NC absorption coefficient $\mu_{\mathrm{i}}$ according to

$$
\varepsilon_{\mathrm{at}}=\frac{f}{\ln (10) \cdot c_{\mathrm{at}}} \mu_{\mathrm{i}}
$$

where $f$ is related to $c_{\text {at }}$ via the $\mathrm{CdSe} / \mathrm{CdS}$ unit cell volume ${ }^{39}$ (24.88 $\AA^{3}$ for WZ CdS and $28.06 \AA^{3}$ for WZ CdSe, respectively). The molar extinction coefficient per NC $\varepsilon_{\mathrm{NC}}$ can be obtained by multiplying $\varepsilon_{\text {at }}$ by the number of atoms per NC. The latter can be determined for core and shell separately, from the ratio of the core (shell) volume and the unit cell volume of $\mathrm{CdSe}(\mathrm{CdS})$.

Finite Element Simulations. The MG EMA predicts that the absorption coefficient of colloidal ( $h$ )NCs suspended in a (transparent) host differs from bulk material due to a dielectric screening of the incident electric field. The polarization of the dielectric (h)NC modifies the internal electric field and thus the effective (h)NC absorption. To accurately calculate this effect, we performed 3D numerical simulations based on a commercially available finite element package (COMSOL Multiphysics) where the scattered field formulation was employed to examine the near field optical properties of CdS NCs. The simulation domain was composed of a spherical volume representing a CdS NC embedded in chloroform with a plane wave as external excitation $(\lambda=295 \mathrm{~nm})$. Perfectly matched layer (PML) boundary conditions were adopted to avoid undesired reflections from the system boundaries. The refractive indices of chloroform and $\mathrm{CdS}$ were taken from Samoc $^{40}$ and Ninomiya, ${ }^{37}$ respectively. Simulations were performed on prolate spheroids and on more realistic representations of the nanorods, that is, cylinders capped by two hemispheres, all with varying aspect ratio. The spheroids had a minor axis length of $1.5 \mathrm{~nm}$ and a major axis between 1.5 and $37.5 \mathrm{~nm}$, and the same dimensions were used for the nanorods (diameter $3 \mathrm{~nm}$, length $75 \mathrm{~nm}$ ). We explicitly took into account the influence of the optical constants of the organic ligand shell on the overall dielectric response, by including an additional dielectric layer around the NCs. The absorption cross section of the CdS NC was calculated by evaluating the portion of incoming light absorbed and transformed into heat by the NC. ${ }^{41,42}$ More specifically, with COMSOL, we evaluated the quantity $1 / 2 \operatorname{Re}\left(J \cdot E^{*}\right)$, which represents the electromagnetic loss ( $J$ : current density, $E$ : electric field). This term was integrated over the NC volume and normalized to the intensity of the incoming radiation to provide the absorption cross section. Finally, the NC absorption coefficient was obtained by dividing the cross section by the NC volume.

Spectroscopic Ellipsometry (SE). The refractive index of HPA was determined by SE. A Si substrate (intrinsic, (111) oriented) was cleaned with piranha solution to remove organic residues. HPA was heated to about $40{ }^{\circ} \mathrm{C}$ and dispersed in ethanol, obtaining a $10 \mathrm{mM}$ solution that was spin-coated on the Si substrate at $4000 \mathrm{rpm}$ for $2 \mathrm{~min}$. During the first minute, the solution was continuously added to ensure complete surface coverage. ${ }^{43} \mathrm{SE}$ data were collected at two angles of incidence $\left(60^{\circ}\right.$ and $\left.65^{\circ}\right)$ on both the HPA-coated and the reference substrate, with a rotating compensator spectroscopic ellipsometer (M-2000, J.A. Woolam Co.Inc.) in the 245-1000 nm wavelength range. The reference substrate was modeled as $\mathrm{Si}$ with an ultrathin native oxide layer, using the optical constants obtained from WVASE software, as done in previous work. ${ }^{44}$ 


\section{RESULTS AND DISCUSSION}

We analyzed the absorption coefficient of three CdSe NC samples with diameters ranging from 3.0 to $4.3 \mathrm{~nm}$, and one $\mathrm{CdS}$ spherical NC sample, with a $4.1 \mathrm{~nm}$ diameter. In addition, we used three $\mathrm{CdSe} / \mathrm{CdS}$ DIDs with varying core sizes and $\mathrm{CdSe} / \mathrm{CdS}$ volume ratios and $12 \mathrm{CdSe} / \mathrm{CdS}$ DIRs with different core sizes and aspect ratios ranging from 2.3 to 21.3. Figure $1 \mathrm{a}-\mathrm{c}$ shows TEM images of representative samples, with

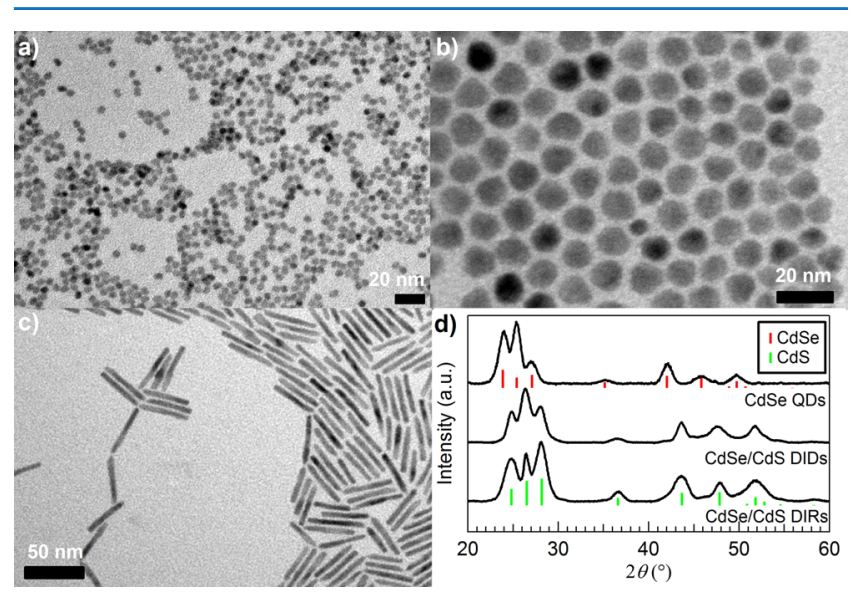

Figure 1. (a) CdSe NCs with a diameter of $4.3 \mathrm{~nm}$; (b) CdSe/CdS DIDs (core diameter $3.2 \mathrm{~nm}$, total diameter $10.5 \mathrm{~nm}$ ); (c) CdSe/CdS DIRs (core diameter $3.5 \mathrm{~nm}$, rod diameter $4.8 \mathrm{~nm}$, rod length 37.9 $\mathrm{nm})$; (d) XRD patterns of representative samples for the three structures. From top to bottom, CdSe cores (6.5 nm diameter), CdSe/ CdS DIDs (core diameter $3.2 \mathrm{~nm}$, total diameter $10.5 \mathrm{~nm}$ ), and CdSe/ CdS DIRs (core diameter: $5.7 \mathrm{~nm}$, rod diameter: $6.2 \mathrm{~nm}$, rod length: $26 \mathrm{~nm}$ ). A WZ crystal structure is observed in all cases. The expected peaks for WZ CdSe and CdS are marked with vertical lines. For the DIRs, the long axis can be identified with the $c$-axis of the crystal from the narrower line width of the [002] peak at $26.5^{\circ} 2 \theta$.

an overview table of all samples analyzed provided in the Supporting Information. Panels (a) and (b) reveal that the NCs are isotropic and fairly monodisperse, with only few smaller particles observed for CdSe/CdS DIDs in panel (b). Also, the anisotropic DIRs (c) have a well-defined and uniform shape. The WZ crystal phase was verified by XRD (Figure 1d). The peaks were attributed to either a WZ CdSe phase (CdSe NCs, PDF card No. 01-075-5681) or a WZ CdS phase (DIRs and DIDs, PDF card No. 00-041-1049) due to the low CdSe volume fraction. Results agree with previous assessments of the $\mathrm{CdSe} / \mathrm{CdS} \mathrm{hNC}$ structure using XRD and high-resolution TEM (HRTEM). ${ }^{15,38}$ The WZ crystal structure constitutes the starting point for the optical analysis, as it determines the optical constants and defines a unique axis in the anisotropic DIRs. More specifically, data confirm that the extraordinary optical axis ( $c$-axis in $\mathrm{WZ} \mathrm{CdSe} / \mathrm{CdS}$ ) coincides with the geometric long axis of the NCs, assigning the different optical constants in $\mathrm{WZ} \mathrm{CdSe}$ and $\mathrm{CdS}$ to directions parallel and perpendicular to the $\mathrm{NC}$ long axis.

For several materials it has already been shown that at energies well above the band gap an intrinsic absorption coefficient $\mu_{\mathrm{i}}$ can be defined ${ }^{39}$ (quasi-)independent of the NC dimensions and only proportional to $f$ (see also eq 1 and the Beer-Lambert law).

$$
\mu_{\mathrm{i}}=\frac{\ln (10) \cdot A}{f \cdot L}
$$

The $\mu_{\mathrm{i}}$ spectra for three typical WZ CdSe NC samples dispersed in chloroform are shown in Figure 2a (solid lines).
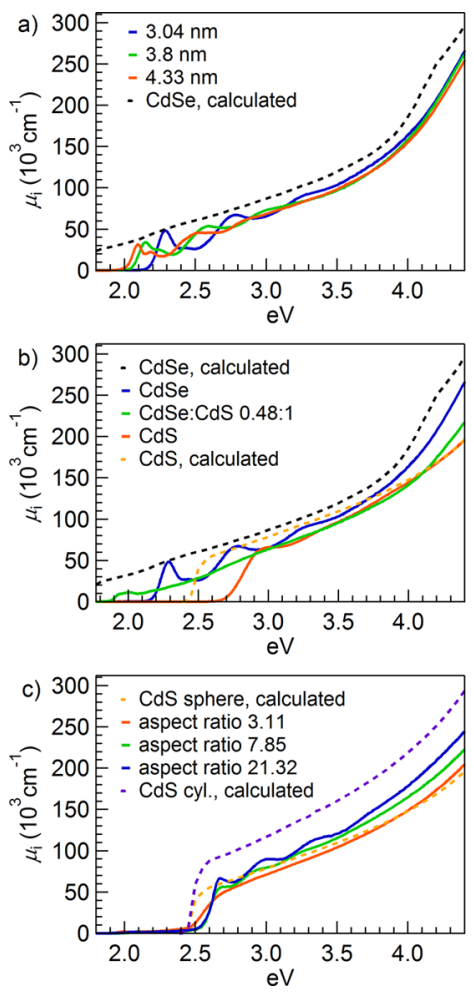

Figure 2. NC absorption coefficient $\mu_{\mathrm{i}}$ in chloroform. (a) For CdSe NCs, experimental $\mu_{\mathrm{i}}$ spectra converge at high energies (solid lines). Dashed line: spectrum calculated with bulk optical constants in the MG EMA. (b) Dependence of $\mu_{\mathrm{i}}$ on the CdSe volume fraction. At high energy the spectra of $\mathrm{CdSe} / \mathrm{CdS}$ core/shell NCs (solid lines) fall between the CdSe and CdS bulk $\mu_{\mathrm{i}}$ (dashed lines, CdSe (blue) and CdS (red)). (c) Aspect ratio-dependence of $\mu_{\mathrm{i}}$. The absorption of CdSe/CdS DIRs is enhanced when aspect ratio increases. To facilitate comparison, we have chosen samples with similar core diameter (3.2$3.5 \mathrm{~nm}$ ). Dashed lines: $\mu_{\mathrm{i}}$ spectra of CdS spheres (red) and infinite cylinders (blue) calculated with bulk optical constants.

Spectra converge above $3.5 \mathrm{eV}$, similar to observations on $\mathrm{WZ}$ CdSe NCs by Jasieniak et al. ${ }^{27}$ and on ZB CdSe NCs by Čapek et $\mathrm{al}^{34}$ However, they also remain slightly below the absorption coefficient calculated within MG EMA ${ }^{45}$ using anisotropic bulk optical constants ${ }^{36}$ (dashed line).

In $\mathrm{CdSe} / \mathrm{CdS}$ hNCs the relative volume fractions of core and shell need to be taken into account for $\mu_{\mathrm{i}}$. Figure $2 \mathrm{~b}$ shows that the $\mu_{\mathrm{i}}$ of CdSe/CdS DIDs falls between the two limiting values of pure $\mathrm{CdSe}$ and CdS. In DIRs, the absorption is further influenced by the aspect ratio. With respect to their spherical counterparts, dielectric screening is strongly suppressed along the NC long axis, leading to enhanced absorption for longer DIRs (Figure 2c).

A quantitative assessment of the optical properties in different $\mathrm{CdSe} / \mathrm{CdS} \mathrm{NCs}$ is performed at $4.2 \mathrm{eV}$. First, we observed that the variation of the CdSe/CdS DID absorption between limiting values of pure $\mathrm{CdSe}$ and $\mathrm{CdS}$ is approximately linear, with absolute values in line with data obtained on $\mathrm{ZB}$ $\mathrm{CdSe} / \mathrm{CdS}{ }^{46}$ In general, in concentric heterostructures the different dielectric constants of core and shell lead to a nonlinear variation of the absorption with volume fraction, ${ }^{47}$ as for instance demonstrated in the case of $\mathrm{PbS} / \mathrm{CdS}$ hNCs. ${ }^{48}$ 
However, here CdSe and CdS have similar optical constants, hence no significant deviation from a linear behavior is observed, in agreement with the linear interpolation described by Park et al. ${ }^{13}$ (see also Supporting Information, Figure S1, for the full analytical calculation).

This dependence allows us to formulate a convenient general model function for $\mu_{\mathrm{i}}$ in hNCs, which becomes a linear combination of the $\mathrm{CdS}$ and $\mathrm{CdSe}$ intrinsic absorption coefficients, simply scaled by their respective volume fractions $f_{\mathrm{CdS}}$ and $f_{\mathrm{CdS}}$. Considering that the CdSe cores are spherical and the CdS shell shape ranges from spherical to rod-like, for $\mu_{\mathrm{CdS}}$, we include a more elaborate expression including the influence of aspect ratio. We can write, with $\mu_{\mathrm{CdS}, \mathrm{dot}}$ and $\mu_{\mathrm{CdS}, \text { rod }}$ the spherical and cylindrical limits of $\mu_{\mathrm{CdS}}$, respectively, and $\mathrm{AR}$ the aspect ratio:

$$
\begin{aligned}
\mu\left(A R, f_{\mathrm{CdS}}\right)= & \left(1-f_{\mathrm{CdS}}\right) \times \mu_{\mathrm{CdSe}} \\
& +f_{\mathrm{CdS}} \times\left\{\mu_{\mathrm{CdS}, \text { rod }}+\left(\mu_{\mathrm{CdS}, \mathrm{dot}}-\mu_{\mathrm{Cd}, \text { rod }}\right)\right. \\
& \times \exp [c(1-\mathrm{AR})]\}
\end{aligned}
$$

While the analytical aspect ratio-dependence according to the MG EMA is given by a more complex expression (see Supporting Information, section 4), we again calculated that the approximation by an exponential aspect ratio-dependence, with $c=0.274$, describes the trend equally well and provides a more practical expression (less than $2 \%$ deviation between the two, see Figure S2). Keeping $c$ fixed, the remaining parameters are determined from a single fit on the whole data set of CdSe, $\mathrm{CdS}, \mathrm{CdSe} / \mathrm{CdS}$ DIDs, and anisotropic DIRs, yielding $\mu_{\mathrm{CdSe}}=$ $(2.05 \pm 0.06) \times 10^{5} \mathrm{~cm}^{-1}, \mu_{\mathrm{CdS}, \mathrm{dot}}=(1.58 \pm 0.06) \times 10^{5} \mathrm{~cm}^{-1}$, and $\mu_{\mathrm{CdS}, \text { rod }}=(2.02 \pm 0.05) \times 10^{5} \mathrm{~cm}^{-1}$. In Figure $3 \mathrm{a}$, the result
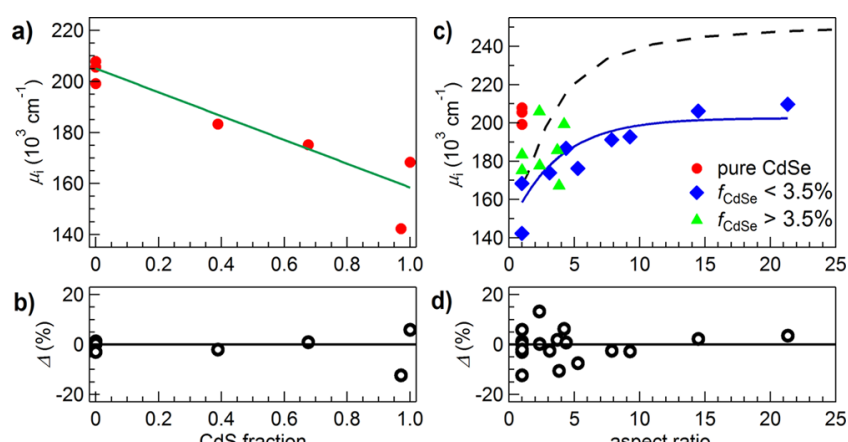

Figure 3. (a) Experimental $\mu_{\mathrm{i}}$ for DIDs with varying CdS volume fraction (red dots). The green line shows the linear transition from pure CdSe to CdS, with relative deviation $\Delta=\left(\mu_{\mathrm{i}, \exp }-\mu_{\mathrm{i}, \mathrm{fit}}\right) / \mu_{\mathrm{i} \text {,exp }}$ plotted in panel (b). (c) Experimental $\mu_{\mathrm{i}}$ for CdSe NCs (red dots), and $\mathrm{CdSe} / \mathrm{CdS}$ NCs with $f_{\mathrm{CdSe}}<3.5 \%$ (blue diamonds) and $f_{\mathrm{CdSe}}>3.5 \%$ (green triangles). Full blue line represents the model in the $f_{\text {CdSe }} \ll 1$ limit, while the dashed line shows the calculated $\mu_{\mathrm{i}}$ using bulk CdS optical constants. The relative deviation $\Delta$ is again shown in panel (d).

of the model is shown for NCs with aspect ratio $=1$ (top, green line). In Figure 3c, experimental data with CdSe core volumes smaller than $3.5 \%$ are also accurately represented by the model in the limit of $f_{\mathrm{CdSe}} \ll 1$ (full blue line). The other samples cannot be represented by a single trend due to a dependence on both aspect ratio and $f_{\mathrm{CdS}}$; however, it is clear that the majority of samples deviate less than $10 \%$ from the empirical fit (Figure $3 b, d$ ), confirming the accuracy of our approach.
Note that from $\mu_{\mathrm{i}}$ one can calculate a molar extinction coefficient $\varepsilon_{\mathrm{NC}}$ using (1), if the total NC volume $V_{\mathrm{NC}}$ and $\mathrm{CdSe} / \mathrm{CdS}$ volume fraction are known so that the number of atoms per NC $N_{\mathrm{at}}$ can be calculated $\left(\varepsilon_{\mathrm{NC}}=\varepsilon_{\mathrm{at}} \times N_{\mathrm{at}}\right){ }^{39}$ Furthermore, the single-particle absorption cross section $\sigma_{\mathrm{NC}}$ is given by $\mu_{\mathrm{i}} \times V_{\mathrm{NC}}$; with typical values of $1.47 \times 10^{-13}$ and 2.27 $\times 10^{-13} \mathrm{~cm}^{2}$ in, for instance, $5 \mathrm{~nm} \times 40 \mathrm{~nm}$ DIRs and $14 \mathrm{~nm}$ "giant-shell” DIDs, respectively, calculated values are in good agreement with literature data ${ }^{13,49}$ (e.g., using the calculation of the $\mathrm{CdSe} / \mathrm{CdS}$ cross section of Park et al., ${ }^{13}$ one finds $1.2 \times$ $10^{-13} \mathrm{~cm}^{2}$ for $14 \mathrm{~nm}$ DIDs at $400 \mathrm{~nm}$, in good agreement with our data at $295 \mathrm{~nm}$ considering an estimated factor of 2 due to the different wavelength used) and serve as a valuable benchmark for further optical spectroscopy or fabrication of photonic devices, as knowledge of absorption coefficient is essential for a better understanding of carrier dynamics, ${ }^{50}$ Auger recombination, $^{51}$ optical gain, ${ }^{52}$ single-dot spectroscopy, ${ }^{53}$ multiexciton and excited state emission ${ }^{50}$ or conversion of light into electrical or electrochemical energy. ${ }^{54}$

While we were able to derive a versatile expression for the absorption of colloidal $\mathrm{CdSe} / \mathrm{CdS}$ hNCs of varying composition and shape, from a theoretical viewpoint the experimental data are still overestimated by the MG EMA when using bulk optical constants. For instance, taking the bulk CdS optical constants and approximating $\mathrm{CdS}$ rods as prolate spheroids suspended in chloroform, we find a typical overestimation of about 20\% (Figure 3c, black dashed line) compared to the experimental trend (blue solid line, NCs in $f_{\text {CdSe }} \ll 1$ limit). This can be related to several aspects: the real NC shape being closer to a cylinder capped by two hemispheres, the organic ligand shell providing an additional dielectric layer, or CdS and CdSe having nonbulk optical constants. To determine their relative contributions to the observed deviation, we calculated the electric field profiles and absorption coefficients of $\mathrm{CdS}$ prolate spheroids, of realistic nanorods, and nanorods surrounded by a ligand shell. In a dielectric inclusion that is exposed to an external electric field $\boldsymbol{E}_{0}$, a polarization $\boldsymbol{P}$ builds up that screens $E_{0}$ and leads to a local electric field $E_{\text {loc }}$ inside the particle. The resulting volume-averaged polarization $\boldsymbol{P}_{\text {avg }}=$ $\int \boldsymbol{P d} V$ can then be related to the absorption cross section and absorption coefficient. ${ }^{55}$ In our case, due to the symmetry of the NC models, $\boldsymbol{P}$ and thus $\boldsymbol{P}_{\text {avg }}$ is parallel to $\boldsymbol{E}_{0}$ (see also Supporting Information, Figure S3), so it suffices to calculate $\mu_{\|}$ and $\mu_{\perp}$, the absorption coefficients of the NC when exposed to an external field parallel and perpendicular to the NC long axis. $\mu_{\mathrm{i}, \text { avg }}=\left(\mu_{\|}+2 \mu_{\perp}\right) / 3$ then yields the orientation-averaged absorption coefficient, whose values can be compared to our experimental data.

As is well-known, the dielectric response of spheroids can be found analytically, and values have been calculated for different eccentricities, random orientations, or multiphase mixtures. ${ }^{56,57}$ For shapes other than ellipsoids, the solution of the electrostatic problem cannot be given in a closed form, and a numerical calculation is needed. ${ }^{58-60} \mathrm{We}$ performed finite element simulations to compare the dielectric properties of CdS nanorods to the ones of spheroids with the same aspect ratios; simulated values of $E_{\text {loc }}$ are shown in Figure 4 for both shapes (aspect ratio $=7$ ) when the electric field is (a) parallel and (b) perpendicular to the NC long axis, with a detailed image of the electric field at the tips in panels (c) and (d). We can readily observe that the electric field toward the tips is somewhat reduced in the nanorods compared to the spheroids. However, this is compensated for by a larger field in the center 

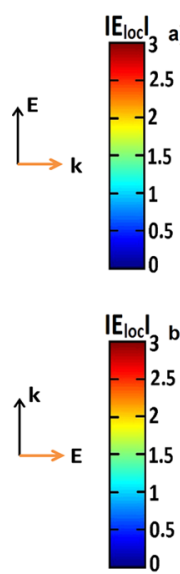
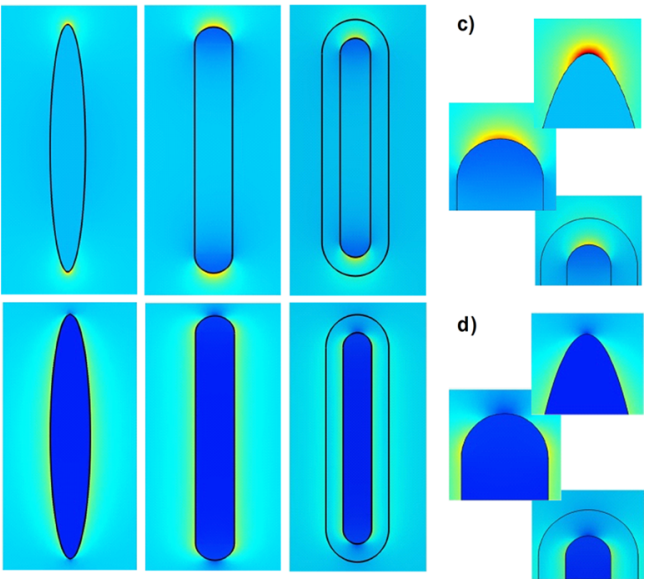

Figure 4. Electric field magnitude $\left|E_{\text {loc }}\right|$ inside the $\mathrm{NC}$ as a result of an applied external field $E_{0}=1 \mathrm{~V} / \mathrm{m}$ at $\lambda=295 \mathrm{~nm}(4.2 \mathrm{eV})$, simulated for spheroids and hemisphere-capped nanorods with and without a ligand shell, when $E_{0}$ is parallel (a) or perpendicular (b) to the NC long axis (aspect ratio $=7$ ). Details of the tip are shown in panels $(c)$ and (d).

of the rod, where its shape is more comparable to an infinitely long cylinder. The resulting $\mu_{\|}$and $\mu_{\perp}$ are shown for the two shapes in Figure 5a for varying aspect ratios (green and black lines). Interestingly, the corresponding $\mu_{\mathrm{i}, \text { avg }}$ for the rod differs by less than $2.5 \%$ from the values simulated for spheroids (Figure $5 \mathrm{~b}$ ). We can thus conclude that the approximation of
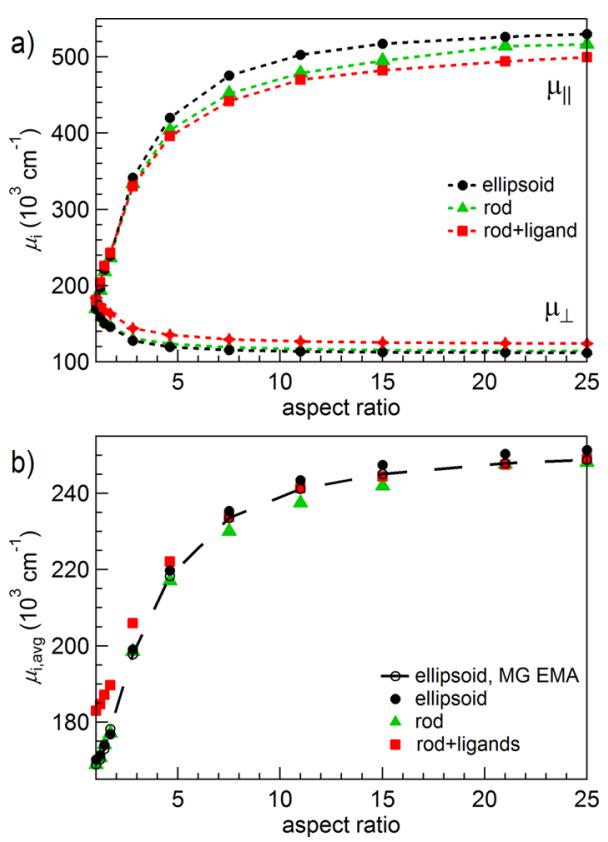

Figure 5. (a) Absorption coefficient of CdS NCs parallel and perpendicular to the NC long axis, simulated for varying aspect ratios of spheroids (black lines), nanorods (green lines), and nanorods surrounded by a ligand shell (red lines). (b) Average absorption coefficient $\mu_{\mathrm{i}, \text { avg }}$ for the three shapes (same markers). Black dashed line shows the absorption coefficient calculated for spheroids within the MG EMA. Values are in good agreement with the finite element simulations of prolate spheroids. Between different simulations, no significant difference is observed for spheroids and nanorods, while an increase in absorption is observed at low aspect ratios when the nanorod is surrounded by a ligand shell. our nanorods as prolate spheroids is justified, and the exact shape does not play a major role for the absorption around 4.2 $\mathrm{eV}$.

The NCs form a stable dispersion in chloroform due to the organic ligand shell, which also contributes to the optical response via its dielectric constant. Regarding the role of organic ligands, difficulties in matching experimental results with the MG EMA had already been reported in previous works. For instance, for CdSe NCs, Leatherdale et al. ${ }^{26}$ discuss that agreement with theory is achieved only when NCs were suspended in hexane, with the added observation that the NC absorption is rather insensitive to changes in the solvent refractive index. Also Jasieniak et al. ${ }^{27}$ found good agreement with bulk only when using the refractive index of hexane, despite the measurements being performed in chloroform. To estimate the dielectric role of the ligand shell, it is necessary to know its refractive index at the wavelength of interest. From here on, we focus on the NC cores and CdSe/CdS DIRs that are capped by HPA or OPDA. ${ }^{61}$ Literature data of an ODPA self-assembled monolayer (SAM) yield a refractive index of $1.61-1.62 .{ }^{62}$ On the other hand, to the best of our knowledge, no data on HPA is reported at present. Therefore, we performed SE on an ultrathin HPA film deposited by spin coating on a silicon substrate. The data were analyzed according to a simple, nondispersive Cauchy model (Figure S4). Since the index of refraction $n_{\text {lig }}$ and the thickness $d$ of a transparent thin dielectric film are correlated, we evaluated the mean square error (MSE) corresponding to different $(n, d)$ pairs, finding a well-defined minimum MSE of $5.65 \pm 0.05$ for $n$ $=1.63 \pm 0.03$ and $d=3.32 \pm 0.05$. On the basis of these data, we performed a numerical simulation adding a $1.8 \mathrm{~nm}$ shell around the CdS nanorods, with $n_{\text {lig }}=1.6$. The resulting $E_{\text {loc }}$ is shown in Figure 4. With $n_{\text {lig }}$ intermediate between both the complex refractive indices of $\mathrm{CdS}(n=2.67, k=0.69)$ and chloroform $\left(n_{\mathrm{s}}=1.49\right)$, we observed an increase in the absorption coefficient for nearly spherical NCs (Figure 5, red and green markers, respectively). Strikingly, at high aspect ratio the presence of the ligands does not significantly modify the overall absorption coefficient. This can be understood from the dominant contribution of $\mu_{\|}$to $\mu_{\mathrm{i}, \text { avg }}$, for which dielectric screening, and thus the influence of an additional ligand layer, is reduced. Nonetheless, independent of the aspect ratio, the ligands again do not fully account for the discrepancies between experimental and theoretical data.

These considerations leave the NC optical constants as remaining variables to explain the difference between experiment and theory. The optical constants of colloidal NCs and their modification due to quantum confinement have already been addressed via pseudopotential calculations, ${ }^{63}$ and experimentally with thin film ellipsometry ${ }^{64,65}$ and KramersKronig analysis of the absorption spectra of suspended NCs. ${ }^{66,67}$ The latter two experimental techniques each have their drawbacks however. Modeling of the SE data requires an effective medium model that accurately takes into account the morphology of the thin film, while the Kramers-Kronig analysis explicitly assumed a continuation of the complex refractive index to high photon energy using bulk values, which, as shown here and in literature, ${ }^{27,35,34}$ is not strictly applicable to Cd-based NCs. Here we present a novel method that takes advantage of the unique aspect ratio-dependence of the dielectric screening, which yields two equations for $\mu_{i}$ in the low- and high-aspect ratio limit. Considering an inorganic semiconductor core coated by an organic ligand shell, according 
to the MG EMA the analytical expression for the polarizabilities $\alpha_{\|}$and $\alpha_{\perp}$ of a coated spheroid excited by an incident field parallel and perpendicular to the NC long axis, respectively, are given by ${ }^{55}$

$$
\begin{aligned}
\alpha_{\|}= & \left\{V \left(\left(\varepsilon_{\text {lig }}-\varepsilon_{\mathrm{s}}\right)\left[\varepsilon_{\text {lig }}+\left(\varepsilon_{\mathrm{CdS}, \|}-\varepsilon_{\text {lig }}\right)\left(L_{\|}-f_{\text {core }} L_{\|}\right)\right]\right.\right. \\
& \left.\left.+f_{\text {core }} \varepsilon_{\text {lig }}\left(\varepsilon_{\mathrm{CdS}, \|}-\varepsilon_{\text {lig }}\right)\right)\right\} /\left\{\left[\varepsilon_{\text {lig }}+\left(\varepsilon_{\mathrm{CdS}, \|}-\varepsilon_{\text {lig }}\right)\left(L_{\|}-f_{\text {core }} L_{\|}\right)\right]\right. \\
& {\left.\left[\varepsilon_{\mathrm{s}}+\left(\varepsilon_{\text {lig }}-\varepsilon_{\mathrm{s}}\right) L_{\|}\right]+f_{\text {core }} L_{\|} \varepsilon_{\text {lig }}\left(\varepsilon_{\mathrm{CdS}, \|}-\varepsilon_{\text {lig }}\right)\right\} } \\
\alpha_{\perp}= & \left\{V \left(\left(\varepsilon_{\text {lig }}-\varepsilon_{\mathrm{s}}\right)\left[\varepsilon_{\text {lig }}+\left(\varepsilon_{\mathrm{CdS}, \perp}-\varepsilon_{\text {lig }}\right)\left(L_{\perp}-f_{\text {core }} L_{\perp}\right)\right]\right.\right. \\
& \left.\left.+f_{\text {core }} \varepsilon_{\text {lig }}\left(\varepsilon_{\mathrm{CdS}, \perp}-\varepsilon_{\text {lig }}\right)\right)\right\} \\
& /\left\{\left[\varepsilon_{\text {lig }}+\left(\varepsilon_{\mathrm{CdS}, \perp}-\varepsilon_{\text {lig }}\right)\left(L_{\perp}-f_{\text {core }} L_{\perp}\right)\right]\left[\varepsilon_{\mathrm{s}}+\left(\varepsilon_{\text {lig }}-\varepsilon_{\mathrm{s}}\right) L_{\perp}\right]\right. \\
& \left.+f_{\text {core }} L_{\perp} \varepsilon_{\text {lig }}\left(\varepsilon_{\mathrm{CdS}, \perp}-\varepsilon_{\text {lig }}\right)\right\}
\end{aligned}
$$

$V$ is the total volume of the particle, $\varepsilon_{\text {lig }}=n_{\text {lig }}{ }^{2}$ is the permittivity of the ligand shell, $\varepsilon_{s}=n_{\mathrm{s}}^{2}$ is the permittivity of the solvent, $f_{\text {core }}$ is the fraction of $V$ occupied by the semiconductor NC, $L_{\|}$and $L_{\perp}$ are the depolarization factor of the $\mathrm{NC}$ along the longitudinal and transversal axis, respectively, and $\varepsilon_{\mathrm{CdS}, \|}$ and $\varepsilon_{\mathrm{CdS}, \perp}$ are the associated complex dielectric function of $\mathrm{CdS}$ at the wavelength of interest. From $\alpha, \mu_{\mathrm{i}, \text { avg }}$ can be obtained as

$$
\mu_{\mathrm{i}, \mathrm{avg}}=\frac{2 \pi n_{\mathrm{s}}}{\lambda V} \operatorname{Im}\left\{\frac{2 \alpha_{\perp}}{3}+\frac{\alpha_{\|}}{3}\right\}
$$

The geometry-dependent depolarization factor $L$ yields two independent equations for $\mu_{\mathrm{i}, \text { avg }}$ in the limiting cases of a sphere and an infinitely long cylinder: $L_{\|}=L_{\perp}=1 / 3$ for the former, while for the latter $L_{\|}=0$ and $L_{\perp}=0.5$ (see also Supporting Information, section 4.1).

Equation 3 already provided an accurate trend for the HPA/ ODPA capped NCs (Figure 3d), considering the experimental values for $\mu_{\mathrm{CdS} \text {,dot }}$ and $\mu_{\mathrm{CdS} \text {,rod }}$ obtained from the empirical fit. Assuming a constant ratio between parallel and perpendicular components of $\varepsilon_{\mathrm{CdS}}$, we then solved eq 5 for both a sphere and an infinitely long cylinder to find $\varepsilon_{\mathrm{r}, \|}=5.9 \pm 0.6, \varepsilon_{\mathrm{r}, \perp}=5.8 \pm$ $0.6, \varepsilon_{\mathrm{i}, \|}=2.7 \pm 0.2$, and $\varepsilon_{\mathrm{i}, \perp}=2.6 \pm 0.2$. By modifying the optical constants of CdS from their bulk values, we finally obtain excellent agreement with experimental data (Figure 6a). Hence, the reduced absorption observed for CdSe/CdS hNCs can be related to a confinement-induced reduction and possible shift of the peaks around 4-5.5 eV in the $\varepsilon_{\mathrm{i}}$ spectrum, which in turn also slightly reduces $\varepsilon_{\mathrm{r}}$ in this spectral region (Figure $6 \mathrm{~b}$ ). It has to be pointed out that as-determined optical constants may hold some uncertainty; we find that, by applying the calculation described, the $2-4 \%$ error on the absorption coefficients translates into an $8-10 \%$ error on the optical constants. More importantly, however, our results highlight a need for further research on the NC optical constants, and the proposed calculations present a unique avenue to obtain them by exploiting the NC shape. As the method is general, further analysis of other NC materials can yield a similar quantification of the optical constants from the absorbance spectra, giving direct information on quantum confined transitions in this spectral region, as well as valuable data to calculate the NC optical properties. Such data could then also be used to accurately model the optical constants and optical properties of close-packed thin films ${ }^{68}$ or NC-doped polymers of glasses. ${ }^{69}$ However, for thin films, at present, highly precise results are missing in literature, as there is no consensus yet on which EMA applies. For instance, Grinolds et al. ${ }^{64}$ reported a
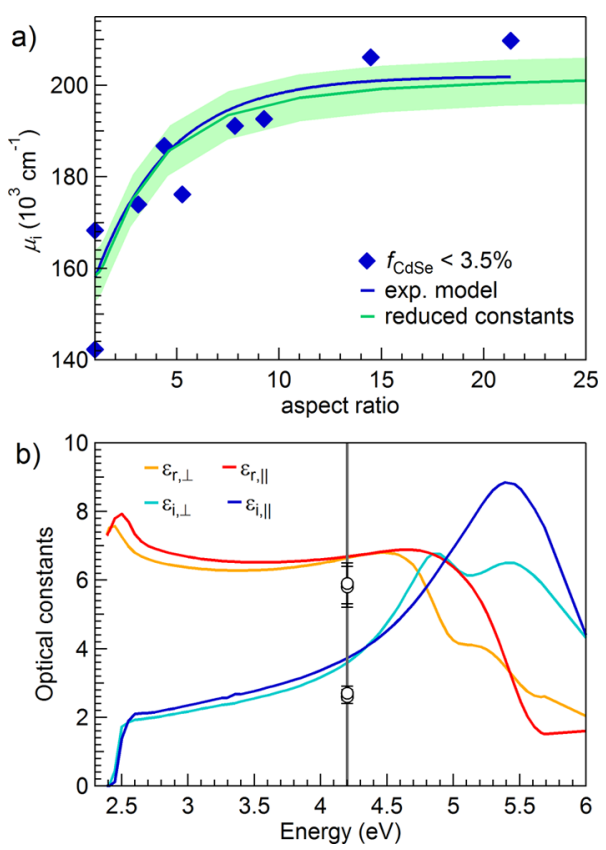

Figure 6. (a) $\mu_{\mathrm{i}}$ of CdSe/CdS NCs with $f_{\text {CdSe }}<3.5 \%$ (blue diamonds). Excellent agreement with experimental values is obtained within the MG EMA when $\varepsilon_{\mathrm{r}}$ and $\varepsilon_{\mathrm{i}}$ are reduced with respect to bulk values (full green line). Calculations using the reduced optical constants are in good agreement with the experimental model function (full blue line, eq 3). The shadowed area represents the range of absorption coefficients obtained taking into account the error on the obtained optical constants. (b) Comparison of the reduced optical constants at $4.2 \mathrm{eV}$ to the bulk CdS optical constants. ${ }^{37}$

consistent mismatch of the dielectric measurements on $\mathrm{PbS}$ thin films with several EMAs. Geiregat et al., ${ }^{70}$ on the other hand, suggested that the MG EMA should be replaced with a coupled dipole model, as dipole coupling in neighboring NCs can significantly enhance the $\mathrm{NC}$ absorption. The issue is also apparent from data of Law et al. ${ }^{65}$ and, more recently, Diroll et al., ${ }^{71}$ who determined the complex refractive index of NC thin films through ellipsometry. While this approach yields precise effective optical constants, in the absence of an accurate EMA, the intrinsic NC optical constants were not obtained. Hence, starting from the determination of the intrinsic NC absorption coefficient and optical constants using the MG EMA in dilute suspensions, a future challenge still lies with the development of accurate thin film effective medium models that can be applied to colloidal NCs.

\section{CONCLUSIONS}

We combined ICP-OES with TEM imaging and absorption spectroscopy to study the absorption coefficient of colloidal CdSe/CdS hNCs. The analysis of both spherical and anisotropic NCs, with compositions ranging from pure $\mathrm{CdSe}$ to pure $\mathrm{CdS}$ and aspect ratios from 1 to 21 , allowed us to obtain a general expression that yields the absorption coefficient of NCs in solution using only the aspect ratio and the $\mathrm{CdSe} / \mathrm{CdS}$ volume ratio. For several systems ("giant-shell" hNCs and most DIRs), an approximation for vanishing core volume can be applied, further simplifying the analysis. We foresee that, considering the similar optical constants of wurtzite and zinc blende $\mathrm{CdX}(\mathrm{X}=\mathrm{S}, \mathrm{Se})$ semiconductors, expressions can be easily extrapolated. A comparison of experimental results with analytical and finite-element numer- 
ical calculations of the absorption coefficient revealed that quantum confinement effects are still present at photon energies well above the band gap. A more realistic nanorod shape did not introduce significant changes to the NC absorption coefficient, and the inclusion of an organic ligand shell influenced the absorption only in the limit of low aspect ratio. This required a deeper understanding of the optical constants of the NCs themselves. In this respect, the unique aspect ratio dependence of $\mu_{\mathrm{i}}$ allowed deriving the NC $\varepsilon_{\mathrm{r}}$ and $\varepsilon_{\mathrm{j}}$, showing that quantitative agreement is reached when both are reduced compared to bulk values. Our method allows for the evaluation of the NC optical constants directly from the absorbance spectra of a set of samples with different aspect ratios, without the need to resort to Kramers-Kronig analysis, ${ }^{6,67}$ or model the optical constants from close-packed thin films. ${ }^{64,65}$ Our results provide a convenient means to a fast, precise, and nondestructive estimate of NC concentrations in solution and constitute an important starting point for further studies addressing the role of shape, crystal structure, and organic shell in the optical properties of colloidal hNCs.

\section{ASSOCIATED CONTENT}

\section{S Supporting Information}

The Supporting Information is available free of charge on the ACS Publications website at DOI: 10.1021/acsphotonics.5b00626.

Details regarding the structural properties of the analyzed samples, finite-element simulations of the polarization density in nanorods, spectroscopic ellipsometry, analytical aspect ratio dependence of $\mu_{\mathrm{i}}$ according to MG EMA, and details on the fitting procedure (PDF).

\section{AUTHOR INFORMATION}

\section{Corresponding Author}

*E-mail: iwan.moreels@iit.it.

\section{Notes}

The authors declare no competing financial interest.

\section{ACKNOWLEDGMENTS}

The present publication is realized with the support of the Ministero degli Affari Esteri e della Cooperazione Internazionale (IONX-NC4SOL, I.M.). The authors thank M. Prato for helpful discussions.

\section{REFERENCES}

(1) Smith, A. M.; Nie, S. Semiconductor Nanocrystals: Structure, Properties, and Band Gap Engineering. Acc. Chem. Res. 2010, 43, 190200.

(2) Lo, S. S.; Mirkovic, T.; Chuang, C.-H.; Burda, C.; Scholes, G. D. Emergent Properties Resulting from Type-II Band Alignment in Semiconductor Nanoheterostructures. Adv. Mater. 2011, 23, 180-197.

(3) Lesnyak, V.; Gaponik, N.; Eychmüller, A. Colloidal Semiconductor Nanocrystals: The Aqueous Approach. Chem. Soc. Rev. 2013, 42, 2905-2929.

(4) Bouet, C.; Tessier, M. D.; Ithurria, S.; Mahler, B.; Nadal, B.; Dubertret, B. Flat Colloidal Semiconductor Nanoplatelets. Chem. Mater. 2013, 25, 1262-1271.

(5) Grim, J. Q.; Manna, L.; Moreels, I. A Sustainable Future for Photonic Colloidal Nanocrystals. Chem. Soc. Rev. 2015, 44, 58975914.

(6) Li, J. J.; Wang, Y. A.; Guo, W.; Keay, J. C.; Mishima, T. D.; Johnson, M. B.; Peng, X. Large-Scale Synthesis of Nearly Monodisperse CdSe/CdS Core/Shell Nanocrystals Using Air-Stable
Reagents via Successive Ion Layer Adsorption and Reaction. J. Am. Chem. Soc. 2003, 125, 12567-12575.

(7) Chen, O.; Zhao, J.; Chauhan, V. P.; Cui, J.; Wong, C.; Harris, D. K.; Wei, H.; Han, H.-S.; Fukumura, D.; Jain, R. K.; et al. Compact High-Quality CdSe-CdS Core-shell Nanocrystals with Narrow Emission Linewidths and Suppressed Blinking. Nat. Mater. 2013, 12, 445-451.

(8) Mahler, B.; Spinicelli, P.; Buil, S.; Quelin, X.; Hermier, J.-P.; Dubertret, B. Towards Non-Blinking Colloidal Quantum Dots. Nat. Mater. 2008, 7, 659-664.

(9) Chen, Y.; Vela, J.; Htoon, H.; Casson, J. L.; Werder, D. J.; Bussian, D. A.; Klimov, V. I.; Hollingsworth, J. A. Giant" Multishell CdSe Nanocrystal Quantum Dots with Suppressed Blinking. J. Am. Chem. Soc. 2008, 130, 5026-5027.

(10) Galland, C.; Ghosh, Y.; Steinbrück, A.; Hollingsworth, J. A.; Htoon, H.; Klimov, V. I. Lifetime Blinking in Nonblinking Nanocrystal Quantum Dots. Nat. Commun. 2012, 3, 908.

(11) García-Santamaría, F.; Chen, Y.; Vela, J.; Schaller, R. D.; Hollingsworth, J. A.; Klimov, V. I. Suppressed Auger Recombination in "Giant" Nanocrystals Boosts Optical Gain Performance. Nano Lett. 2009, 9, 3482-3488.

(12) Zhao, J.; Chen, O.; Strasfeld, D. B.; Bawendi, M. G. Biexciton Quantum Yield Heterogeneities in Single CdSe (CdS) Core (Shell) Nanocrystals and Its Correlation to Exciton Blinking. Nano Lett. 2012, 12, 4477-4483.

(13) Park, Y.-S.; Malko, A. V.; Vela, J.; Chen, Y.; Ghosh, Y.; GarcíaSantamaría, F.; Hollingsworth, J. A.; Klimov, V. I.; Htoon, H. NearUnity Quantum Yields of Biexciton Emission from $\mathrm{CdSe} / \mathrm{CdS}$ Nanocrystals Measured Using Single-Particle Spectroscopy. Phys. Rev. Lett. 2011, 106, 187401.

(14) Talapin, D. V.; Koeppe, R.; Götzinger, S.; Kornowski, A.; Lupton, J. M.; Rogach, A. L.; Benson, O.; Feldmann, J.; Weller, H. Highly Emissive Colloidal CdSe/CdS Heterostructures of Mixed Dimensionality. Nano Lett. 2003, 3, 1677-1681.

(15) Carbone, L.; Nobile, C.; De Giorgi, M.; Sala, F. D.; Morello, G.; Pompa, P.; Hytch, M.; Snoeck, E.; Fiore, A.; Franchini, I. R.; et al. Synthesis and Micrometer-Scale Assembly of Colloidal CdSe/CdS Nanorods Prepared by a Seeded Growth Approach. Nano Lett. 2007, 7, 2942-2950.

(16) Talapin, D. V.; Nelson, J. H.; Shevchenko, E. V.; Aloni, S.; Sadtler, B.; Alivisatos, A. P. Seeded Growth of Highly Luminescent $\mathrm{CdSe} / \mathrm{CdS}$ Nanoheterostructures with Rod and Tetrapod Morphologies. Nano Lett. 2007, 7, 2951-2959.

(17) Allione, M.; Ballester, A.; Li, H.; Comin, A.; Movilla, J. L.; Climente, J. I.; Manna, L.; Moreels, I. Two-Photon-Induced Blue Shift of Core and Shell Optical Transitions in Colloidal CdSe/CdS QuasiType II Quantum Rods. ACS Nano 2013, 7, 2443-2452.

(18) Wu, K.; Rodríguez-Córdoba, W. E.; Liu, Z.; Zhu, H.; Lian, T. Beyond Band Alignment: Hole Localization Driven Formation of Three Spatially Separated Long-Lived Exciton States in CdSe/CdS Nanorods. ACS Nano 2013, 7, 7173-7185.

(19) Moreels, I.; Rainò, G.; Gomes, R.; Hens, Z.; Stöferle, T.; Mahrt, R. F. Nearly Temperature-Independent Threshold for Amplified Spontaneous Emission in Colloidal CdSe/CdS Quantum Dot-in-Rods. Adv. Mater. 2012, 24, OP231-OP235.

(20) Schlamp, M. C.; Peng, X.; Alivisatos, A. P. Improved Efficiencies in Light Emitting Diodes Made with $\mathrm{CdSe}(\mathrm{CdS})$ Core/shell Type Nanocrystals and a Semiconducting Polymer. J. Appl. Phys. 1997, 82, $5837-5842$.

(21) Achtstein, A. W.; Hennig, J.; Prudnikau, A.; Artemyev, M. V.; Woggon, U. Linear and Two-Photon Absorption in Zero- and OneDimensional CdS Nanocrystals: Influence of Size and Shape. J. Phys. Chem. C 2013, 117, 25756-25760.

(22) Dai, X.; Zhang, Z.; Jin, Y.; Niu, Y.; Cao, H.; Liang, X.; Chen, L.; Wang, J.; Peng, X. Solution-Processed, High-Performance LightEmitting Diodes Based on Quantum Dots. Nature 2014, 515, 96-99.

(23) Pisanello, F.; Martiradonna, L.; Leménager, G.; Spinicelli, P.; Fiore, A.; Manna, L.; Hermier, J.-P.; Cingolani, R.; Giacobino, E.; 
Vittorio, M. D.; et al. Room Temperature-Dipolelike Single Photon Source with a Colloidal Dot-in-Rod. Appl. Phys. Lett. 2010, 96, 033101. (24) Grivas, C.; Li, C.; Andreakou, P.; Wang, P.; Ding, M.; Brambilla, G.; Manna, L.; Lagoudakis, P. Single-Mode Tunable Laser Emission in the Single-Exciton Regime from Colloidal Nanocrystals. Nat. Commun. 2013, 4, 2376.

(25) Xing, G.; Chakrabortty, S.; Chou, K. L.; Mishra, N.; Huan, C. H. A.; Chan, Y.; Sum, T. C. Enhanced Tunability of the Multiphoton Absorption Cross-Section in Seeded CdSe/CdS Nanorod Heterostructures. Appl. Phys. Lett. 2010, 97, 061112.

(26) Leatherdale, C. A.; Woo, W.-K.; Mikulec, F. V.; Bawendi, M. G. On the Absorption Cross Section of CdSe Nanocrystal Quantum Dots. J. Phys. Chem. B 2002, 106, 7619-7622.

(27) Jasieniak, J.; Smith, L.; van Embden, J.; Mulvaney, P.; Califano, M. Re-Examination of the Size-Dependent Absorption Properties of CdSe Quantum Dots. J. Phys. Chem. C 2009, 113, 19468-19474.

(28) Kamal, J. S.; Gomes, R.; Hens, Z.; Karvar, M.; Neyts, K.; Compernolle, S.; Vanhaecke, F. Direct Determination of Absorption Anisotropy in Colloidal Quantum Rods. Phys. Rev. B: Condens. Matter Mater. Phys. 2012, 85, 035126.

(29) Brus, L. Zero-Dimensional "Excitons" in Semiconductor Clusters. IEEE J. Quantum Electron. 1986, 22, 1909-1914.

(30) Ekimov, A. I.; Hache, F.; Schanne-Klein, M. C.; Ricard, D.; Flytzanis, C.; Kudryavtsev, I. A.; Yazeva, T. V.; Rodina, A. V.; Efros, A. L. Absorption and Intensity-Dependent Photoluminescence Measurements on CdSe Quantum Dots: Assignment of the First Electronic Transitions. J. Opt. Soc. Am. B 1993, 10, 100.

(31) Ricard, D.; Ghanassi, M.; Schanne-Klein, M. C. Dielectric Confinement and the Linear and Nonlinear Optical Properties of Semiconductor-Doped Glasses. Opt. Commun. 1994, 108, 311-318.

(32) Klimov, V. I. Optical Nonlinearities and Ultrafast Carrier Dynamics in Semiconductor Nanocrystals. J. Phys. Chem. B 2000, 104, 6112-6123.

(33) Moreels, I.; Lambert, K.; De Muynck, D.; Vanhaecke, F.; Poelman, D.; Martins, J. C.; Allan, G.; Hens, Z. Composition and SizeDependent Extinction Coefficient of Colloidal PbSe Quantum Dots. Chem. Mater. 2007, 19, 6101-6106.

(34) Čapek, R. K.; Moreels, I.; Lambert, K.; De Muynck, D.; Zhao, Q.; Van Tomme, A.; Vanhaecke, F.; Hens, Z. Optical Properties of Zincblende Cadmium Selenide Quantum Dots. J. Phys. Chem. C 2010, 114, 6371-6376.

(35) Kamal, J. S.; Omari, A.; Van Hoecke, K.; Zhao, Q.; Vantomme, A.; Vanhaecke, F.; Capek, R. K.; Hens, Z. Size-Dependent Optical Properties of Zinc Blende Cadmium Telluride Quantum Dots. J. Phys. Chem. C 2012, 116, 5049-5054.

(36) Ninomiya, S.; Adachi, S. Optical Properties of Cubic and Hexagonal CdSe. J. Appl. Phys. 1995, 78, 4681-4689.

(37) Ninomiya, S.; Adachi, S. Optical Properties of Wurtzite CdS. J. Appl. Phys. 1995, 78, 1183-1190.

(38) Christodoulou, S.; Vaccaro, G.; Pinchetti, V.; De Donato, F.; Grim, J. Q.; Casu, A.; Genovese, A.; Vicidomini, G.; Diaspro, A.; Brovelli, S.; et al. Synthesis of Highly Luminescent Wurtzite CdSe/ CdS Giant-Shell Nanocrystals Using a Fast Continuous Injection Route. J. Mater. Chem. C 2014, 2, 3439.

(39) Hens, Z.; Moreels, I. Light Absorption by Colloidal Semiconductor Quantum Dots. J. Mater. Chem. 2012, 22, 10406.

(40) Samoc, A. Dispersion of Refractive Properties of Solvents: Chloroform, Toluene, Benzene, and Carbon Disulfide in Ultraviolet, Visible, and near-Infrared. J. Appl. Phys. 2003, 94, 6167-6174.

(41) Alabastri, A.; Toma, A.; Malerba, M.; De Angelis, F.; Proietti Zaccaria, R. High Temperature Nanoplasmonics: The Key Role of Nonlinear Effects. ACS Photonics 2015, 2, 115-120.

(42) Knight, M. W.; Halas, N. J. Nanoshells to Nanoeggs to Nanocups: Optical Properties of Reduced Symmetry Core-shell Nanoparticles beyond the Quasistatic Limit. New J. Phys. 2008, 10, 105006 .

(43) Nie, H.-Y.; Walzak, M. J.; McIntyre, N. S. Delivering Octadecylphosphonic Acid Self-Assembled Monolayers on a Si
Wafer and Other Oxide Surfaces. J. Phys. Chem. B 2006, 110, 21101-21108.

(44) Toccafondi, C.; Cavalleri, O.; Bisio, F.; Canepa, M. Yeast Cytochrome c Adsorption on $\mathrm{SiO} 2 / \mathrm{Si}$ Substrates Studied by in Situ Spectroscopic Ellipsometry. Thin Solid Films 2013, 543, 78-82.

(45) Sihvola, A. Two Main Avenues Leading To the Maxwell Garnett Mixing Rule. Journal of Electromagnetic Waves and Applications 2001, $15,715-725$.

(46) Omari, A. Optical Properties of Colloidal Quantum Dot Functionalized Silicon-On-Insulator Waveguides. Ph.D. Thesis, Universiteit Gent, 2015.

(47) Neeves, A. E.; Birnboim, M. H. Composite Structures for the Enhancement of Nonlinear-Optical Susceptibility. J. Opt. Soc. Am. B $1989,6,787$.

(48) Justo, Y.; Geiregat, P.; Hoecke, K. V.; Vanhaecke, F.; De Mello Donega, C.; Hens, Z. Optical Properties of PbS/CdS Core/Shell Quantum Dots. J. Phys. Chem. C 2013, 117, 20171-20177.

(49) Saba, M.; Minniberger, S.; Quochi, F.; Roither, J.; Marceddu, M.; Gocalinska, A.; Kovalenko, M. V.; Talapin, D. V.; Heiss, W.; Mura, A.; et al. Exciton-Exciton Interaction and Optical Gain in Colloidal CdSe/CdS Dot/Rod Nanocrystals. Adv. Mater. 2009, 21, 4942-4946.

(50) Galland, C.; Brovelli, S.; Bae, W. K.; Padilha, L. A.; Meinardi, F.; Klimov, V. I. Dynamic Hole Blockade Yields Two-Color Quantum and Classical Light from Dot-in-Bulk Nanocrystals. Nano Lett. 2013, 13, 321-328.

(51) García-Santamaría, F.; Brovelli, S.; Viswanatha, R.; Hollingsworth, J. A.; Htoon, H.; Crooker, S. A.; Klimov, V. I. Breakdown of Volume Scaling in Auger Recombination in CdSe/CdS Heteronanocrystals: The Role of the Core-Shell Interface. Nano Lett. 2011, 11, 687-693.

(52) Klimov, V. I.; Ivanov, S. A.; Nanda, J.; Achermann, M.; Bezel, I.; McGuire, J. A.; Piryatinski, A. Single-Exciton Optical Gain in Semiconductor Nanocrystals. Nature 2007, 447, 441-446.

(53) Galland, C.; Ghosh, Y.; Steinbrück, A.; Sykora, M.; Hollingsworth, J. A.; Klimov, V. I.; Htoon, H. Two Types of Luminescence Blinking Revealed by Spectroelectrochemistry of Single Quantum Dots. Nature 2011, 479, 203-207.

(54) Meinardi, F.; Colombo, A.; Velizhanin, K. A.; Simonutti, R.; Lorenzon, M.; Beverina, L.; Viswanatha, R.; Klimov, V. I.; Brovelli, S. Large-Area Luminescent Solar Concentrators Based on "Stokes-ShiftEngineered" Nanocrystals in a Mass-Polymerized PMMA Matrix. Nat. Photonics 2014, 8, 392-399.

(55) Bohren, C. F.; Huffman, D. R. Introduction. Absorption and Scattering of Light by Small Particles; Wiley-VCH Verlag GmbH, 1998.

(56) Sihvola, A. H.; Kong, J. A. Effective Permittivity of Dielectric Mixtures. IEEE Transactions on Geoscience and Remote Sensing 1988, 26, 420-429.

(57) Giordano, S. Effective Medium Theory for Dispersions of Dielectric Ellipsoids. J. Electrost. 2003, 58, 59-76.

(58) Venermo, J.; Sihvola, A. Dielectric Polarizability of Circular Cylinder. J. Electrost. 2005, 63, 101-117.

(59) Sihvola, A.; Yla-Oijala, P.; Jarvenpaa, S.; Avelin, J. Polarizabilities of Platonic Solids. IEEE Trans. Antennas Propag. 2004, 52, 2226-2233.

(60) Mejdoubi, A.; Brosseau, C. Finite-Element Simulation of the Depolarization Factor of Arbitrarily Shaped Inclusions. Phys. Rev. E 2006, 74, 031405.

(61) Gomes, R.; Hassinen, A.; Szczygiel, A.; Zhao, Q.; Vantomme, A.; Martins, J. C.; Hens, Z. Binding of Phosphonic Acids to CdSe Quantum Dots: A Solution NMR Study. J. Phys. Chem. Lett. 2011, 2, $145-152$.

(62) Nie, H.-Y.; McIntyre, N. S.; Lau, W. M.; Feng, J. M. Optical Properties of an Octadecylphosphonic Acid Self-Assembled Monolayer on a Silicon Wafer. Thin Solid Films 2008, 517, 814-818.

(63) Wang, L.-W.; Zunger, A. Pseudopotential Calculations of Nanoscale CdSe Quantum Dots. Phys. Rev. B: Condens. Matter Mater. Phys. 1996, 53, 9579-9582.

(64) Grinolds, D. D. W.; Brown, P. R.; Harris, D. K.; Bulovic, V.; Bawendi, M. G. Quantum-Dot Size and Thin-Film Dielectric 
Constant: Precision Measurement and Disparity with Simple Models. Nano Lett. 2015, 15, 21-26.

(65) Law, M.; Beard, M. C.; Choi, S.; Luther, J. M.; Hanna, M. C.; Nozik, A. J. Determining the Internal Quantum Efficiency of PbSe Nanocrystal Solar Cells with the Aid of an Optical Model. Nano Lett. 2008, 8, 3904-3910.

(66) Alves-Santos, M.; Felice, R. D.; Goldoni, G. Dielectric Functions of Semiconductor Nanoparticles from the Optical Absorption Spectrum: The Case of CdSe and CdS. J. Phys. Chem. C 2010, 114, 3776-3780.

(67) Moreels, I.; Allan, G.; De Geyter, B.; Wirtz, L.; Delerue, C.; Hens, Z. Dielectric Function of Colloidal Lead Chalcogenide Quantum Dots Obtained by a Kramers-Krönig Analysis of the Absorbance Spectrum. Phys. Rev. B: Condens. Matter Mater. Phys. 2010, $81,235319$.

(68) Sandeep, C. S. S.; Azpiroz, J. M.; Evers, W. H.; Boehme, S. C.; Moreels, I.; Kinge, S.; Siebbeles, L. D. A.; Infante, I.; Houtepen, A. J. Epitaxially Connected PbSe Quantum-Dot Films: Controlled Neck Formation and Optoelectronic Properties. ACS Nano 2014, 8, 1149911511.

(69) Moreels, I.; Kruschke, D.; Glas, P.; Tomm, J. W. The Dielectric Function of PbS Quantum Dots in a Glass Matrix. Opt. Mater. Express 2012, 2, 496.

(70) Geiregat, P.; Justo, Y.; Abe, S.; Flamee, S.; Hens, Z. Giant and Broad-Band Absorption Enhancement in Colloidal Quantum Dot Monolayers through Dipolar Coupling. ACS Nano 2013, 7, 987-993.

(71) Diroll, B. T.; Gaulding, E. A.; Kagan, C. R.; Murray, C. B. Spectrally-Resolved Dielectric Functions of Solution-Cast Quantum Dot Thin Films. Chem. Mater. 2015, 27, 6463-6469. 